\title{
Development and validation of RP-HPLC method for pitavastatin calcium in bulk and formulation using experimental design
}

\author{
Vinodkumar D. Ramani ${ }^{1 *}$, Girish K. Jani², Ashim Kumar Sen ${ }^{3}$, Girish U. Sailor ${ }^{3}$, Vijaykumar B. Sutariya ${ }^{4}$ \\ ${ }^{1}$ Pharmacy Department, Gujarat Technological University, Chandkheda, Ahmedabad 382424, India. \\ ${ }^{2}$ K. B. Raval College of Pharmacy, Kasturi Nagar, Gandhinagar 382423, India. \\ ${ }^{3}$ Department of Pharmacy, Sumandeep Vidyapeeth, Piparia, Vadodara 391760, India. \\ ${ }^{4}$ USF College of Pharmacy, University of South Florida Health, MDC 30, Tampa, FL 33612-4749, USA.
}

\section{ARTICLE INFO \\ Received on: 23/01/2019 \\ Accepted on: 02/05/2019 \\ Available online: 05/10/2019}

\section{Key words:}

Pitavastatin calcium,

RP-HPLC, design of experiment, mobile phase optimization, simplex

centroid design.

\begin{abstract}
The optimization of HPLC method involves several variables whose influence has been widely studied. However, in most of the cases, only process variables are taken into account. In this work, the influence of mixture composition on peak quality parameters of Pitavastatin calcium in bulk and tablet dosage form has been studied using a mixture simplex design. A simplex centroid design with axial points in a pseudo-component representation was generated from the pure mixture components. Twelve ternary mixture mobile phases corresponding to augmented design points were tested to separate the drug in sample. The statistical analysis was performed to generate the polynomial equation for each response. The desirability approach was used to determine the optimal mobile phase composition. Furthermore, the method was validated as per the ICH guidelines using specificity, linearity, accuracy, precision, sensitivity, system suitability, and robustness. The results of experimental design were statistically tested for full and in portion to get best fitted model which accurately describe changes in the proportion of these solvents in the mobile phase close to the region of optimal peak quality. The method demonstrated optimum chromatographic separation with isocratic elution of the mobile phase containing a mixture of acetonitrile-water ( $\mathrm{pH} 3.0$ )-tetrahydrofuran (43:55:02, v/v/v) with a flow rate at $1.0 \mathrm{ml} / \mathrm{minute}$. Design of experiment optimization strategy is a powerful tool to acquire the maximum quality data while performing minimum number of experiments. The mobile phase composition was successfully optimized using simplex centroid mixture design with desirability approach. Additionally, developed method can be applied for routine quantitative analysis of Pitavastatin calcium in bulk and tablet dosage form as it was found to be simple, sensitive, and robust.
\end{abstract}

\section{INTRODUCTION}

Pitavastatin, chemically (E)-7- [2-cyclopropyl-4(4-fluorophenyl) quinolin-3-yl]-3,5-dihydroxy- hept-6- enoic acid, is fully synthetic statin and inhibitor of HMG-CoA reductase. Pitavastatin (commonly as a calcium salt) is a more potent antihyperlipidemic agent compared to other statins (Kajinami et al., 2003). The estimation of Pitavastatin calcium (PTV) from pharmaceutical formulations has been conducted using several

\footnotetext{
"Corresponding Author

Vinodkumar D. Ramani, Pharmacy Department, Gujarat Technological University, Chandkheda, Ahmedabad 382424, India.

E-mail: vinod.ramani01@gmail.com
}

analytical methods, including high-performance thin layer chromatography (Akabari et al., 2015; Kumar and Baghyalakshmi 2007; Patel et al., 2011), high performance liquid chromatography (Gomas et al., 2010; Kojima et al., 1999; Panchal et al., 2008), and liquid chromatography-tandem mass spectrometry (Deng et al., 2008; Di et al., 2008). However, there were no reports available in literature to quantify PTV by DoE (design of experiment) approach.

The purpose of optimization strategy is to acquire the maximum quality data while performing a minimum number of experiments. However, in traditional methodology, optimization is carried out by change in one or two parameters deliberately or by trial and error to rule out the relationship among chromatographic parameters. This methodology may become unpredictable and does not take account of possible synergistic effects among variables. Beside this, literature surveys reported the utility of experimental 
design methodology for optimization of mobile phase composition which is more reliable and avoid the limitations of traditional methodology (Beg et al., 2012; Berridge, 1984; Hasnain et al., 2013; Srinubabu et al., 2007). However, the objective of the experiment is mostly taken into consideration for appropriate selection of design. The present work was aimed to improve peak quality parameters by optimizing the mobile phase composition. This problem can be effectively resolved by mixture designs (Alves de Almeida and Spacino Scarminio 2007; Atkinson and Tobias 2008; Cano et al., 2006). There are three types of established mixture designs: simplex-centroid design, simplex lattice design, and axial design. Furthermore, the design should be efficient to optimize inside the constrained simplex mixture space. Therefore, simplex centroid mixture design with axial points in a pseudo-component representation was used in this study. Moreover, the developed method was also subjected to validation as per ICH guidelines.

\section{EXPERIMENTAL}

\section{Chemicals and reagents}

Pitavastatin calcium reference standard was provided as gift sample by Zydus Cadila, India. HPLC grade acetonitrile $(\mathrm{ACN})$ and tetrahydrofuran (THF) were obtained from Merck Life Science Pvt. Ltd., Mumbai, India. The deionized water was obtained from the EMD Millipore Direct-Q 3 System (Millipore Corporation, Bedford, MA).

\section{Instrumentation}

Shimadzu UFLC Prominence (Shimadzu, Kyoto, Japan) equipped with LC-20AD binary pump, PD-M20A PDA detector, and LC solution as a software was used for the analysis. Separation was done using C18 column (Phenomenex kromasil, 250×4.6 mm i.d., $5 \mu \mathrm{m}$ particle size, $100 \AA$ ). The drug and all the analytical chemicals were weighed on an electronic balance (AUW 220, Shimadzu Corporation, Kyoto, Japan).

\section{Mobile phase and sample preparation}

$\mathrm{ACN}$ and THF were taken into a volumetric flask and 3 $\mathrm{pH}$ water $\left(\mathrm{H}_{2} \mathrm{O}\right)$ was used to make up the volume $500 \mathrm{ml}$. The $\mathrm{pH}$ of deionized water was adjusted using $0.1 \% \mathrm{v} / \mathrm{v}$ trifluoroacetic acid.

A stock solution containing $500 \mu \mathrm{g} / \mathrm{ml}$ of PTV was prepared by dissolving in a mobile phase composed of ACN: $\mathrm{H}_{2} \mathrm{O}:$ THF $(33: 34: 33, \mathrm{v} / \mathrm{v} / \mathrm{v})$. The working standard solution containing $1 \mu \mathrm{g} / \mathrm{ml}$ of PTV was prepared from the above stock solution.

To prepare sample stock solution $(500 \mu \mathrm{g} / \mathrm{ml}), 20$ tablets ( 2 mg PTV per tablet; Pivasta ${ }^{\circledR}, \mathrm{mfd}$. by Zydus Cadila, Ahmedabad, India) were weighed and pulverized. Powdered tablet equivalent to $5 \mathrm{mg}$ of PTV was dissolved in mobile phase (5 $\mathrm{ml}$ ) followed by sonication (15 minutes) and volume was made up to $10 \mathrm{ml}$. Placebo solution was also prepared in a similar manner but without PTV in the tablet. The obtained solution was clarified using a membrane filter ( $47 \mathrm{~mm}, 0.45 \mu \mathrm{m})$.

The working sample solution $(5 \mu \mathrm{g} / \mathrm{ml})$ was prepared by diluting $0.1 \mathrm{ml}$ aliquot of stock solution to $10 \mathrm{ml}$ with the mobile phase. Finally, PTV sample solution $(1 \mu \mathrm{g} / \mathrm{ml})$ was prepared by diluting $2 \mathrm{ml}$ aliquot of working sample solution to $10 \mathrm{ml}$ with the mobile phase.

\section{Chromatographic procedure}

The flow rate $(1 \mathrm{ml} / \mathrm{minute})$, injection volume $(20 \mu \mathrm{l})$, and concentration of solution $(1 \mu \mathrm{g} / \mathrm{ml})$ were maintained constant for all the batches. The detection wavelength was selected as 249 $\mathrm{nm}$ based on the absorption maxima of the UV spectrum obtained from chromatographic system (Fig. 1). Identical environmental conditions $\left(25^{\circ} \mathrm{C}\right.$ and $\left.45 \% \mathrm{RH}\right)$ were maintained during all the runs.

\section{Software aided method optimization}

\section{Mixture design study}

The components of mobile phase were selected on the basis of preliminary trials. From the pure mixture components ( $\mathrm{ACN}, \mathrm{H}_{2} \mathrm{O}$, and $\mathrm{THF}$ ), a simplex centroid design with axial points in a pseudo-component representation was generated. The pseudo-components are represented by $X_{1}(48: 50: 2), X_{2}(38: 60: 2)$, and $X_{3}(38: 50: 12)$, different proportions of $\mathrm{ACN}, \mathrm{H}_{2} \mathrm{O}$, and THF, respectively (Fig. 2). The component proportions in each mixture sum to $100 \%$, i.e., volume of $100 \mathrm{ml}$. Table 1 represents the binary and ternary combinations of these pseudo-components.

In the mixture optimization problem, the proportion of the ingredients is assumed to be the only factor responsible for measuring responses. The responses were measured as $Y_{1}$ : Retention time (RT), $Y_{2}$ : Tailing factor (TF), and $Y_{3}$ : Theoretical plates (TP). Each possible mixture compositions of mobile phase (Table 1) was tested one time in random order and center point run was replicated two more times to evaluate the residuals and lack-of-fit.

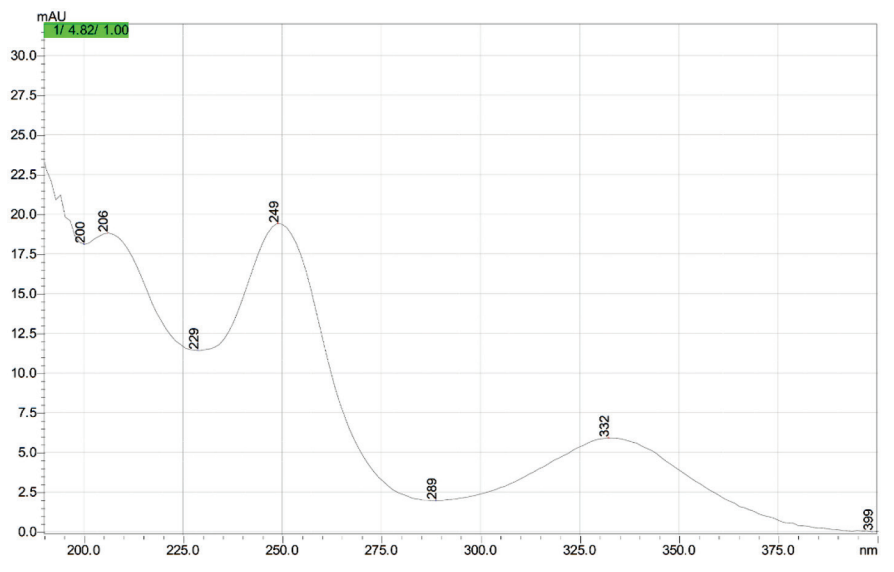

Figure 1. UV spectrum of Pitavastatin calcium.

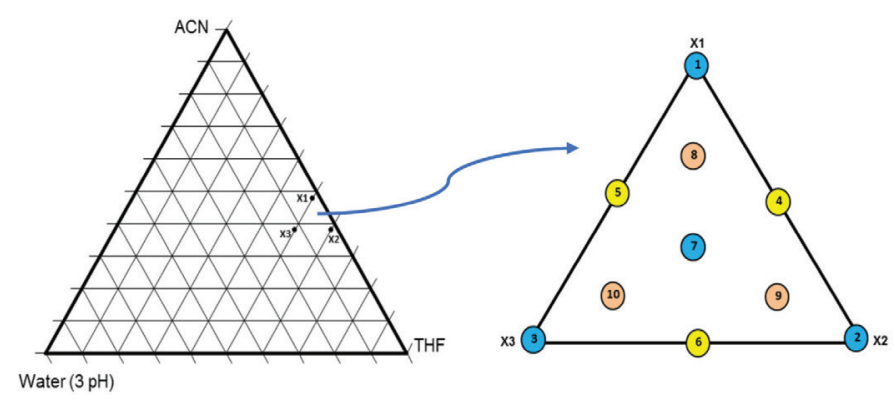

Figure 2. The sub-region of the original mixture simplex design refined as a simplex in the pseudo-components $\left(X_{1}, X_{2}\right.$, and $\left.X_{3}\right)$. 
Table 1. Mobile phase compositions of mixture design and respective responses.

\begin{tabular}{|c|c|c|c|c|c|c|c|c|}
\hline \multirow{2}{*}{ Sr. no. } & \multicolumn{3}{|c|}{ Composition of pseudo-components } & \multirow{2}{*}{$\begin{array}{c}\% \text { composition } \\
\text { ACN: Water: THF }\end{array}$} & \multicolumn{3}{|c|}{ Responses } & \multirow{2}{*}{ Space type } \\
\hline & $X_{1}$ & $X_{2}$ & $X_{3}$ & & $Y_{1}:$ RT & $Y_{2}: \mathrm{TF}$ & $Y_{3}: \mathrm{TP}$ & \\
\hline 1 & 1 & 0 & 0 & $48: 50: 2$ & 4.13 & 1.236 & 6129 & $\mathrm{~V}$ \\
\hline 2 & 0 & 1 & 0 & $38: 60: 2$ & 6.60 & 1.227 & 7699 & $\mathrm{~V}$ \\
\hline 3 & 0 & 0 & 1 & $38: 50: 12$ & 4.97 & 1.205 & 7182 & $\mathrm{~V}$ \\
\hline 4 & 0.5 & 0.5 & 0 & $43: 55: 2$ & 4.99 & 1.287 & 7026 & $\mathrm{CE}$ \\
\hline 5 & 0.5 & 0 & 0.5 & $38: 55: 7$ & 5.64 & 1.188 & 7467 & $\mathrm{CE}$ \\
\hline 6 & 0 & 0.5 & 0.5 & $43: 50: 7$ & 4.52 & 1.22 & 6740 & $\mathrm{CE}$ \\
\hline 7 & 0.333 & 0.333 & 0.333 & $41.33: 53.33: 5.34$ & 5.10 & 1.242 & 7090 & $\mathrm{C}$ \\
\hline 8 & 0.667 & 0.167 & 0.167 & $44.67: 51.66: 3.67$ & 4.48 & 1.243 & 6755 & $\mathrm{ACB}$ \\
\hline 9 & 0.1667 & 0.6667 & 0.1667 & $39.67: 56.66: 3.67$ & 5.65 & 1.255 & 7408 & $\mathrm{ACB}$ \\
\hline 10 & 0.167 & 0.167 & 0.667 & $39.67: 51.66: 8.67$ & 4.98 & 1.203 & 7154 & $\mathrm{ACB}$ \\
\hline 11 & 0.333 & 0.333 & 0.333 & $41.33: 53.33: 5.34$ & 5.07 & 1.254 & 7230 & $\mathrm{C}$ \\
\hline 12 & 0.333 & 0.333 & 0.333 & $41.33: 53.33: 5.34$ & 5.07 & 1.24 & 7143 & $\mathrm{C}$ \\
\hline
\end{tabular}

ACN indicates: Acetonitrile; THF: tetra hydro furan; V: vertex; CE: centers of edges; C: Center; ACB: axial check blends; $Y_{1}$, retention time (RT); $Y_{2}$, tailing factor (TF); $Y_{3}$, theoretical plates (TP); $X_{1},(48: 50: 2) ; X_{2},(38: 60: 2) ; X_{3},(38: 50: 12)$.

\section{Statistical analysis setup}

Simplex design for mixture component optimization can be mathematically represented by Eq. 1, therefore response can be estimated experimentally for any combination of the mixture components (Montgomery, 2008; Rao and Baral, 2011):

$$
\sum_{i=1}^{q} x_{i}=x_{1}+x_{2}+\ldots x_{q}=1 ; x_{i}>0 ; i=1,2,3, \ldots q
$$

where, $q$ denotes the no. of mixture components and $X_{i}$ denotes the fraction of $i$ th component in the mixture. In widespread use, the standard forms of the mixture models are (Cornell, 2011) as follows:

$$
\begin{aligned}
& \text { Linear }: y=\sum_{i=1}^{q} \beta_{i} x_{i} \\
& \text { Quadratic }: y=\sum_{i=1}^{q} \beta_{i} x_{i}+\sum \sum_{i<j}^{q} \beta_{i j} x_{i} x_{i j} \\
& \text { FullCubic: } y=\sum_{i=1}^{q} \beta_{i} x_{i}+\sum \sum_{i<j}^{q} \beta_{i j} x_{i} x_{j} \\
& +\sum \sum_{i<j}^{q} \delta_{i j} x_{i} x_{j}\left(x_{i}-x_{j}\right) \\
& +\sum \sum_{i<j<k}^{q} \beta_{i j k} x_{i} x_{j} x_{k} \\
& \text { SpecialCubic : } y=\sum_{i=1}^{q} \beta_{i} x_{i}+\sum \sum_{i<j}^{q} \beta_{i j} x_{i} x_{j} \\
& +\sum \sum_{i<j<k}^{q} \beta_{i j k} x_{i} x_{j} x_{k}
\end{aligned}
$$

$$
\begin{aligned}
\text { SpecialCubic : } y= & \sum_{i=1}^{q} \beta_{i} x_{i}+\sum \sum_{i<j}^{q} \beta_{i j} x_{i} x_{j}+\sum \sum_{i<j<k}^{q} \beta_{i j k} x_{i}^{2} x_{j} x_{k} \\
& +\sum \sum_{i<j<k}^{q} \beta_{i j k^{k} i} x_{j}^{2} x_{k}+\sum \sum_{i<j<k}^{q} \beta_{i j k_{i}^{*}} x_{j} x_{k}^{2}
\end{aligned}
$$

where, $Y$ denotes the response variable, $\beta_{i}$ denotes the pure blend estimated response, $X_{j}=0$ and $X_{i}=1$ when $i \neq j$. The linear blending portion expressed as $\Sigma_{i=1}^{q} \beta_{i} X_{i}$. The parameters $\beta_{i j}$ represent either antagonistic or synergistic effect arising from the nonlinear blending between component pairs. In mixture models, higher order terms are often essential due to the large operability region and require elaborate model to explain the complex phenomenon.

\section{Check point analysis}

The desirability function approach was used to search for the optimized mixture composition. A selection from suggested mixture composition was done based on ease of mobile phase preparation (No decimal value for each component) and minimum use of THF was desired. Validation was performed by check point analysis. A selected mixture composition was used to analyze the standard solution of PTV $(1 \mu \mathrm{g} / \mathrm{ml})$ and evaluated for responses.

\section{System suitability study}

The system suitability was determined by injecting six replicate of standard solution $(1 \mu \mathrm{g} / \mathrm{ml}$ PTV). Various parameters, such as RT, PA (peak area), TF, and TP, were evaluated.

\section{Method validation}

The method, after development, was validated as per ICH guidelines Q2 (R1) (ICH Guideline, 2010).

\section{Linearity}

To assess the linearity of the proposed method, the concentration range $10-500 \mathrm{ng} / \mathrm{ml}$ was prepared by diluting 0.1 , $0.2,0.5,1,2.5$, and $5 \mathrm{ml}$ aliquots of working standard solution to $10 \mathrm{ml}$ with the mobile phase. Each solution was injected (20 $\mu 1, n=6$ ) and peak area was recorded. The calibration curve was 
constructed by plotting peak areas versus concentrations of PTV. The linearity was expressed as a correlation coefficient by linear regression analysis.

\section{Sensitivity}

The limit of detection (LOD) and the limit of quantification (LOQ) of the drug were calculated using the following equations:

$$
\begin{aligned}
& L O D=3.3 \sigma / S \\
& L O Q=10 \sigma / S
\end{aligned}
$$

where, $S$ and $\sigma$ represents the standard deviation of response and slope, respectively.

\section{Precision}

The sample solution $(100 \mathrm{ng} / \mathrm{ml})$ was injected $(n=6)$ on the same day to assess the repeatability. The intraday precision was performed at three different times in a day (i.e., morning, afternoon, and evening), while inter-day precision was evaluated three times in three consecutive days. The three altered concentrations (50, 100 , and $250 \mathrm{ng} / \mathrm{ml} ; n=3$ ) were used to estimate intraday and inter-day precision. The percentage RSD was calculated from peak area.

\section{Accuracy}

The recovery experiment of PTV was used to find the accuracy of the developed method. The accuracy of the method was determined by calculating recoveries of PTV by the standard addition method. In pre-quantified sample solution $(100 \mathrm{ng} / \mathrm{ml})$, a known amount of standard solutions of PTV $(80,100$, and 120 $\mathrm{ng} / \mathrm{ml}$ ) were added. The quantity of PTV was measured using a calibration curve.

\section{Specificity}

Specificity of developed RP-HPLC method was scrutinized by injecting standard, sample, and placebo solution. They were compared to evaluate the interference between excipients and drug peak.

\section{Robustness}

Robustness of the proposed method was assessed by altering the organic solvent ( $\mathrm{ACN})$ content of the mobile phase, $\mathrm{pH}$ of water in the mobile phase, flow rate, detection wavelength, and extraction time of PTV.

\section{Analysis of marketed formulation}

The sample solution $(1 \mu \mathrm{g} / \mathrm{ml})$ of marketed tablet was prepared as per the method given in "Mobile phase and Sample preparation" section. Sample solution containing $1 \mu \mathrm{g} / \mathrm{ml}$ of PTV was analyzed $(n=6)$ and the average peak area was calculated to determine the drug content in marketed tablet.

\section{RESULTS}

The preliminary investigation suggested that the mobile phase composition acetonitrile-water $(\mathrm{pH}$ 3.0)-tetrahydrofuran showed good chromatographic separation with symmetrical peak and reproducible results.

\section{Optimization of mobile phase composition using simplex centroid design}

\section{Model fitting and regression analysis}

The reasonable impacts of independent variables were observed in all the cases, while performing the experiments in random order (Table 1). The results were fitted to different mixture models and the residual errors were estimated to examine the goodness of fit for each model. The software suggested that the best fitted model was special quartic for $Y_{1}$ and $Y_{3}$, while the quadratic model for $Y_{2}$. The model summary statistics are given in Table 2. The regression coefficients for each of the responses are shown in Table 3. A positive value denoted an effect that favored the optimization, while a negative value indicated an inverse relationship between the factor and the response. The polynomial equation of full model was generated for each response (Eqs. 9-11).

$$
\begin{aligned}
R T= & 4.12 x_{1}+6.59 x_{2}+4.96 x_{3}-1.53 x_{1} x_{2}+4.33 x_{1} x_{3} \\
& -5.09 x_{2} x_{3}-37.15 x_{1}^{2} x_{2} x_{3}+40.61 x_{1} x_{2}^{2} x_{3}+3.53 x_{1} x_{2} x_{3}^{2} \\
T F= & 1.23 x_{1}+1.23 x_{2}+1.20 x_{3}+0.25 x_{1} x_{2}-0.11 x_{1} x_{3} \\
& +0.04 x_{2} x_{3} \\
T P= & 6129.25 x_{1}+7699.25 x_{2}+7182.25 x_{3}+448.99 x_{1} x_{2} \\
& +3246.99 x_{1} x_{3}-2801.01 x_{2} x_{3}-17146.56 x_{1}^{2} x_{2} x_{3} \\
& +23029.44 x_{1} x_{2}^{2} x_{3}-1666.56 x_{1} x_{2} x_{3}^{2}
\end{aligned}
$$

The statistical validity of the polynomial equation was established on the basis of analysis of variance (ANOVA). Table 4 shows the results of ANOVA and lack of fit tests where the $F$-value for $Y_{1}=210.56, Y_{2}=29.92$, and $Y_{3}=67.53$ and the value of $R^{2}$ for $Y_{1}$ $=0.9982, Y_{2}=0.9614$, and $Y_{3}=0.9945$. Values of $p$ less than 0.05 indicates that model terms are significant except for responses $Y_{1}$, model terms $X_{1} X_{2} X^{2}{ }_{3}$ ( $p$ value: 0.5489), for $Y_{2}$, model term $X_{2} X_{3}$ ( $p$ value: 0.2985$)$, and for $Y_{3}$, model term $X_{1} X_{2}(p$ value: 0.210$)$, $X_{1}{ }_{1} X_{2} X_{3}$ ( $p$ value: 0.061$)$, and $X_{1} X_{2} X^{2}{ }_{3}(p$ value: 0.794$)$ showed an essential model reduction to improve the model (Table 3 ).

The generated reduced model was tested by $F$ statistics in portions. The reduced model polynomial equations (Eqs. 1214) were generated for $Y_{1}, Y_{2}$, and $Y_{3}$ :

$$
\begin{aligned}
R T= & 4.12 x_{1}+6.59 x_{2}+4.97 x_{3}-1.52 x_{1} x_{2}+4.36 x_{1} x_{3} \\
& -5.05 x_{2} x_{3}-35.74 x_{1}^{2} x_{2} x_{3}+42.02 x_{1} x_{2}^{2} x_{3} \\
T F= & 1.23 x_{1}+1.23 x_{2}+1.21 x_{3}+0.25 x_{1} x_{2}-0.11 x_{1} x_{3} \\
T P= & 6131.68 x_{1}+7701.68 x_{2}+7178.80 x_{3}+446.95 x_{1} x_{2} \\
& +3233.19 x_{1} x_{3}-2814.81 x_{2} x_{3}-17808.39 x_{1}^{2} x_{2} x_{3} \\
& +22367.61 x_{1} x_{2}^{2} x_{3}
\end{aligned}
$$


Table 2. Model summary statistics for responses.

\begin{tabular}{|c|c|c|c|c|c|}
\hline Responses & Models & SD & $R^{2}$ & Adjusted $R^{2}$ & PRESS \\
\hline \multirow{6}{*}{$Y_{1}: \mathrm{RT}$} & Linear & 0.52 & 0.4710 & 0.3534 & 4.86 \\
\hline & Quadratic & 0.19 & 0.9499 & 0.9081 & 3.75 \\
\hline & Special Cubic & 0.21 & 0.9516 & 0.8935 & 7.20 \\
\hline & Cubic & 0.052 & 0.9982 & 0.9935 & 4.31 \\
\hline & Special Quartic & 0.052 & 0.9982 & 0.9935 & 4.31 \\
\hline & Quartic & 0.017 & 0.9999 & 0.9993 & + \\
\hline \multirow{6}{*}{$Y_{2}: \mathrm{TF}$} & Linear & 0.022 & 0.4675 & 0.3492 & 0.010 \\
\hline & Quadratic & 0.0071 & 0.9614 & 0.9293 & 0.0029 \\
\hline & Special Cubic & 0.0062 & 0.9755 & 0.9462 & 0.0025 \\
\hline & Cubic & 0.0067 & 0.9827 & 0.9364 & 0.014 \\
\hline & Special Quartic & 0.0067 & 0.9827 & 0.9364 & 0.014 \\
\hline & Quartic & 0.0075 & 0.9857 & 0.9211 & + \\
\hline \multirow{6}{*}{$Y_{3}: \mathrm{TP}$} & Linear & 329.23 & 0.4614 & 0.3417 & $1,999,000$ \\
\hline & Quadratic & 108.34 & 0.9611 & 0.9287 & $1,021,000$ \\
\hline & Special Cubic & 116.28 & 0.9627 & 0.9179 & $1,940,000$ \\
\hline & Cubic & 57.74 & 0.9945 & 0.9798 & $24,174.86$ \\
\hline & Special Quartic & 57.74 & 0.9945 & 0.9798 & $24,174.86$ \\
\hline & Quartic & 70.68 & 0.9945 & 0.9697 & + \\
\hline
\end{tabular}

SD indicates: standard deviation; $R^{2}$, regression coefficient; PRESS: predicted residual error sum of squares; $Y_{1}$, retention time (RT); $Y_{2}$, tailing factor (TF); $Y_{3}$, theoretical plates (TP).

Table 3. Summary of results for regression analysis.

\begin{tabular}{|c|c|c|c|c|c|c|c|c|c|c|c|}
\hline \multirow{2}{*}{ Response } & \multirow{2}{*}{ Model } & & \multicolumn{9}{|c|}{ Model term } \\
\hline & & & $X_{1}$ & $X_{2}$ & $X_{3}$ & $\boldsymbol{X}_{1} \boldsymbol{X}_{2}$ & $\boldsymbol{X}_{T} \boldsymbol{X}_{3}$ & $\boldsymbol{X}_{2} \boldsymbol{X}_{3}$ & $\boldsymbol{X}^{2} \boldsymbol{X}_{2} \boldsymbol{X}_{3}$ & $\boldsymbol{X}_{I} \boldsymbol{X}^{2} \boldsymbol{X}_{3}$ & $\boldsymbol{X}_{I} \boldsymbol{X}_{2} \boldsymbol{X}_{3}^{2}$ \\
\hline \multirow{3}{*}{$Y_{1}: \mathrm{RT}$} & \multirow{3}{*}{$\begin{array}{l}\text { Special } \\
\text { Quartic }\end{array}$} & FM & 4.12 & 6.59 & 4.96 & -1.53 & 4.33 & -5.09 & -37.15 & 40.61 & 3.53 \\
\hline & & $p$-value & 0.0002 & & & 0.0092 & 0.0004 & 0.0003 & 0.0058 & 0.0045 & $0.5489^{\#}$ \\
\hline & & $\mathrm{RM}$ & 4.12 & 6.59 & 4.97 & -1.52 & 4.36 & -5.06 & -35.74 & 42.02 & - \\
\hline \multirow{3}{*}{$Y_{2}: \mathrm{TF}$} & \multirow{3}{*}{ Quadratic } & FM & 1.23 & 1.23 & 1.20 & 0.25 & -0.11 & 0.04 & - & - & - \\
\hline & & $p$-value & 0.0004 & & & 0.0002 & 0.0116 & $0.2616^{\#}$ & - & - & - \\
\hline & & $\mathrm{RM}$ & 1.23 & 1.23 & 1.21 & 0.25 & -0.11 & - & & & \\
\hline \multirow{3}{*}{$Y_{3}: \mathrm{TP}$} & \multirow{3}{*}{$\begin{array}{l}\text { Special } \\
\text { Quartic }\end{array}$} & $\mathrm{FM}$ & 6129.25 & 7699.25 & 7182.25 & 448.99 & 3246.99 & -2801.01 & -17146.56 & 23029.44 & -1666.56 \\
\hline & & $p$-value & 0.001 & & & $0.210^{\#}$ & 0.001 & 0.002 & $0.061^{\#}$ & 0.029 & $0.794^{\#}$ \\
\hline & & $\mathrm{RM}$ & 6131.68 & 7701.68 & 7178.80 & 446.95 & 3233.19 & -2814.81 & -17808.39 & 22367.61 & - \\
\hline
\end{tabular}

$Y_{1}$ indicates: retention time (RT); $Y_{2}$, tailing factor (TF); $Y_{3}$, theoretical plates (TP); FM: full model; RM: reduced model; ${ }^{*}$ Not significant $(p>0.05)$.

The 3D surface and contour plots were created using Design-Expert ${ }^{\circledR}$ software for better understanding of the effect of the variables (Fig. 3). The overlaid contour plot was generated as per the desired targets for the optimum response variables represented by yellow zone (Fig. 4).

\section{Optimization}

The desired targets for responses were set to optimize the formulation. Target ranges in response $Y_{1} \geq 4.5, Y_{2} \leq 2$ and $Y_{3} \geq 6,000$. On the basis of this, the software suggested the various compositions of mobile phase starting with the highest desirability value. The optimized mobile phase batch (OC), ACN: $\mathrm{H}_{2} \mathrm{O}$ : THF $(43: 55: 2, \mathrm{v} / \mathrm{v} / \mathrm{v})$, was selected with desirability value 1. These compositions were used for HPLC run and responses were evaluated. Similarly, experimental design and polynomial equations were validated by checkpoint analysis of validation batch, $\mathrm{VC}(38: 50: 12, \mathrm{v} / \mathrm{v} / \mathrm{v})$. The $\%$ bias between predicted and experimental value for $\mathrm{OC}$ and $\mathrm{VC}$ were given in Table 5.

\section{System suitability}

The results of the system suitability test show that the $\%$ RSD of RT, PA, TF, and TP were found to be $0.429,1.919,0.703$, and 1.091, respectively (Table 6).

\section{Method validation}

PTV showed a good correlation in the linearity range of $10-500 \mathrm{ng} / \mathrm{ml}\left(r^{2}=0.9993\right)$ for the developed HPLC method. The linear regression equation was $y=166.54 x+742.43$. Repeatability of the method was found to be $0.893-1.676$ ( $\% \mathrm{RSD}, n=6)$. Intraday and inter-day precision were found to be $0.839-1.534$ ( $\% \mathrm{RSD}, n=3)$ and $0.88-1.405$ ( $\% \mathrm{RSD}, n=3)$, respectively (Table 7). The result of recovery studies, used to assess the accuracy of the developed HPLC method are presented 
Table 4. Calculations for testing the model in portions.

\begin{tabular}{|c|c|c|c|c|c|c|c|c|c|}
\hline & Source & SS & df & MS & $F$ value & $p$-value & $R^{2}$ & $\operatorname{Adj~} R^{2}$ & $F$ statistic \\
\hline \multicolumn{10}{|l|}{$Y_{1}: R T$} \\
\hline \multirow{2}{*}{ Regression } & $\mathrm{FM}$ & 4.54 & 8 & 0.57 & 210.56 & 0.00049 & 0.9982 & 0.9934 & \multirow{6}{*}{$\begin{aligned} F_{\mathrm{Cal}} & =0.444 \\
F_{\mathrm{Tab}} & =10.128 \\
\mathrm{DF} & =(1,3) \\
\alpha & =0.05\end{aligned}$} \\
\hline & $\mathrm{RM}$ & 4.54 & 7 & 0.65 & 278.65 & $<0.0001$ & 0.998 & 0.9944 & \\
\hline \multirow{2}{*}{ Residual } & FM & 0.0081 & 3 & 0.0027 & \multirow{4}{*}{14.52} & \multirow{4}{*}{0.0644} & & & \\
\hline & $\mathrm{RM}$ & 0.0093 & 4 & 0.0023 & & & & & \\
\hline Lack of Fit & & 0.0087 & 2 & 0.0044 & & & & & \\
\hline Pure Error & & 0.0006 & 2 & 0.0003 & & & & & \\
\hline \multicolumn{10}{|l|}{$Y_{2}:$ TF } \\
\hline \multirow{2}{*}{ Regression } & FM & 0.00768 & 5 & 0.001537 & 29.92 & 0.00036 & 0.9614 & 0.9293 & \multirow{6}{*}{$\begin{aligned} F_{\text {Cal }} & =1.569 \\
F_{\text {Tab }} & =5.987 \\
\text { DF } & =(1,6) \\
\alpha & =0.05\end{aligned}$} \\
\hline & $\mathrm{RM}$ & 0.00761 & 4 & 0.001901 & 34.38 & 0.00011 & 0.9516 & 0.9239 & \\
\hline \multirow{2}{*}{ Residual } & FM & 0.00031 & 6 & 0.000051 & \multirow{4}{*}{0.9504} & \multirow{4}{*}{0.5845} & & & \\
\hline & $\mathrm{RM}$ & 0.00039 & 7 & 0.000055 & & & & & \\
\hline Lack of Fit & & 0.00027 & 5 & 0.000054 & & & & & \\
\hline Pure Error & & 0.00011 & 2 & 0.000057 & & & & & \\
\hline \multicolumn{10}{|l|}{$\mathbf{Y}_{3}: \mathbf{T P}$} \\
\hline \multirow{2}{*}{ Regression } & FM & $1,801,172.46$ & 8 & $225,146.56$ & 67.53 & 0.0027 & 0.9945 & 0.9798 & \multirow{6}{*}{$\begin{aligned} F_{\mathrm{Cal}} & =0.081 \\
F_{\text {Tab }} & =10.128 \\
\mathrm{DF} & =(1,3) \\
\alpha & =0.05\end{aligned}$} \\
\hline & $\mathrm{RM}$ & $1,800,900.26$ & 7 & $257,271.47$ & 100.16 & 0.0003 & 0.9943 & 0.9844 & \\
\hline \multirow{2}{*}{ Residual } & FM & $10,001.79$ & 3 & $3,333.93$ & \multirow{4}{*}{0.0282} & \multirow{4}{*}{0.9726} & & & \\
\hline & $\mathrm{RM}$ & $10,273.99$ & 4 & $2,568.50$ & & & & & \\
\hline Lack of Fit & & 281.32 & 2 & 140.66 & & & & & \\
\hline Pure Error & & $9,992.67$ & 2 & $4,996.33$ & & & & & \\
\hline
\end{tabular}

$Y_{1}$ indicates: retention time (RT); $Y_{2}$, tailing factor (TF); $Y_{3}$, theoretical plates (TP); SS, sum of squares; df, degrees of freedom; MS, mean of squares; $F$, Fischer's ratio; $R^{2}$, regression coefficient; FM: full model; RM: reduced model; $F_{\text {Tab }}$, Table value of $F ; F_{\text {Calc }}$, Calculated value of $F$. Details of calculations are shown by Mendenhall W. and Sincich.
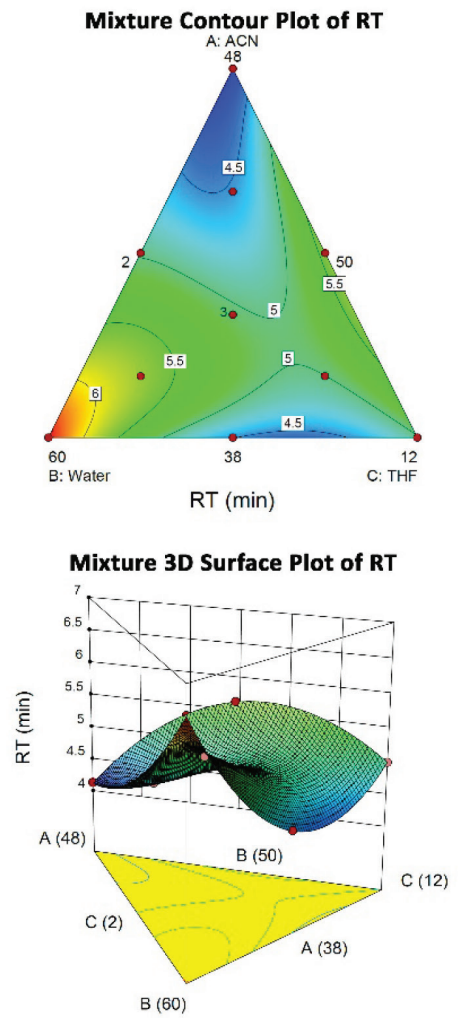
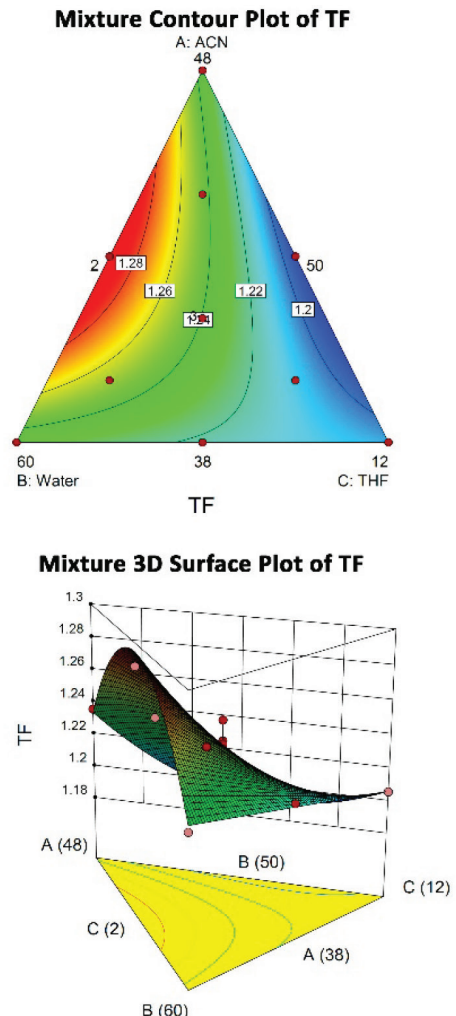

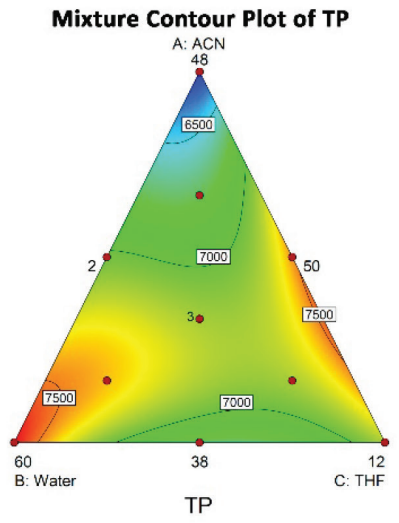

Mixture 3D Surface Plot of TP

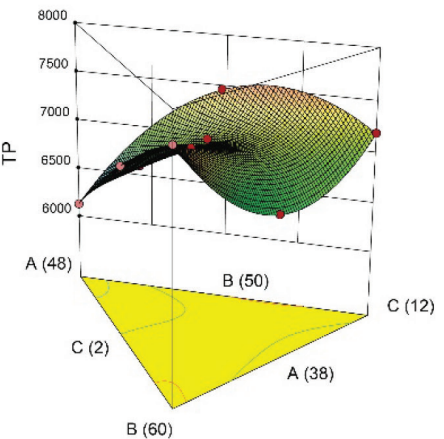

$A: X_{1}(48: 50: 2) ; B: X_{2}(38: 60: 2) ; C: X_{3}(38: 50: 12)$

Figure 3. Contour and 3D surface plot for RT $\left(Y_{1}\right)$, TF $\left(Y_{2}\right)$, and TP $\left(Y_{3}\right)$. 


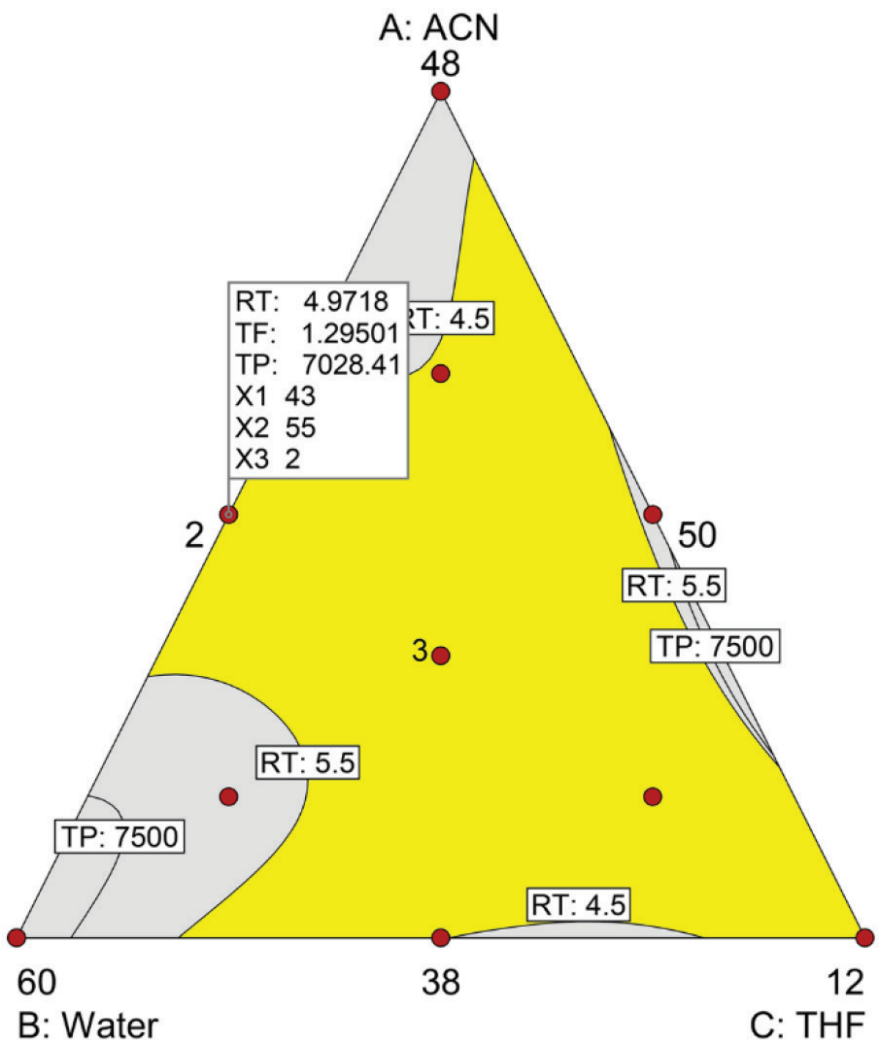

Figure 4. Overlaid contour plot for RT $\left(Y_{1}\right), \operatorname{TF}\left(Y_{2}\right)$, and $\mathrm{TP}\left(Y_{3}\right)$.

Table 5. Check point analysis of experimental design.

\begin{tabular}{llcc}
\hline \multirow{2}{*}{ Responses } & \multirow{2}{*}{$\begin{array}{l}\text { Result } \\
\text { category }\end{array}$} & \multicolumn{2}{c}{ Selected mixture composition (ACN: $\left.\mathbf{H}_{\mathbf{2}} \mathbf{O}: \mathbf{T H F}\right)$} \\
\cline { 3 - 4 } & Predicted & 4.97 & $\mathbf{3 8 : 5 0 : 1 2}(\mathbf{V C})$ \\
\hline \multirow{3}{*}{$Y_{1}: \mathrm{RT}$} & Experimental & 4.82 & 4.97 \\
& \% Bias & 3.01 & 5.43 \\
& Predicted & 1.295 & 8.40 \\
$Y_{2}: \mathrm{TF}$ & Experimental & 1.22 & 1.206 \\
& \% Bias & 5.79 & 1.206 \\
& Predicted & 7028 & 0 \\
$Y_{3}: \mathrm{TP}$ & Experimental & 6881 & 7178 \\
& \% Bias & 2.08 & 7104 \\
\hline
\end{tabular}

$Y_{1}$ indicates: retention time (RT); $Y_{2}$, tailing factor (TF); $Y_{3}$, theoretical plates (TP); CAN: Acetonitrile; THF: tetrahydrofuran; OC: optimized batch composition; VC: validation batch composition.

in Table 7. The LOQ and LOD were found to be 5.907 and 1.949 $\mathrm{ng} / \mathrm{ml}$, respectively. Standard $(1 \mu \mathrm{g} / \mathrm{ml})$, sample $(1 \mu \mathrm{g} / \mathrm{ml})$, and placebo solutions were injected into the HPLC system to check the specificity. The peak purity was found to be $99.99 \%$ and no extra peaks were found within RT around 5 minutes. The result of robustness study shows that the $\%$ RSD of the various selected parameters were found to be less than 2 (Table 8 ).

\section{Analysis of marketed dosage form}

The chromatogram showed the PTV peak at RT $4.82 \pm$ 0.02 minutes, peak area $168,937.17 \pm 3,242.55$, TF $1.22 \pm 0.01$, and TP $6,692.5 \pm 73.02$. The drug content was found to be $99.12 \%$ $(\% \mathrm{RSD} \pm 0.09)$.
Table 6. System suitability parameters.

\begin{tabular}{lcc}
\hline Parameters & Mean \pm SD & \%RSD \\
\hline RT (min) & $4.82 \pm 0.02$ & 0.429 \\
PA & $168,937.17 \pm 3,242.55$ & 1.919 \\
TF & $1.22 \pm 0.01$ & 0.703 \\
TP & $6,692.5 \pm 73.02$ & 1.091 \\
\hline
\end{tabular}

RSD indicates: relative standard deviation; RT: retention time; TF: tailing factor; TP: theoretical plates; PA: peak area reproducibility.

\section{DISCUSSION}

The selection of mobile phase components was based on preliminary investigations and literature survey. Acetonitrile $(\mathrm{ACN})$, methanol $(\mathrm{MeOH})$, and tetrahydrofuran $(\mathrm{THF})$ were tested as the organic phase modifying solvents. These investigations revealed that a change in the organic modifier from $\mathrm{MeOH}$ to $\mathrm{ACN}$, the peak symmetry was improved. Moreover, addition of THF with ACN resulted in sharper peak and enhanced resolution. The finding also showed that $\mathrm{pH}$ adjustment was essential to improve the peak symmetry. The fact that PTV is an acidic compound which requires the mobile phase $\mathrm{pH}$ below its $\mathrm{pKa}$ for better resolution and sharper peak. In order to achieve $3 \mathrm{pH}$ of the aqueous phase, $5 \%$ orthophosphoric acid (OPA), and $1 \%$ trifluoroacetic acid (TFA) were tested. The splitting of peak was observed with 5\% OPA, while 1\% TFA showed sharper peak with adequate resolution.

\section{Optimization of mobile phase composition using simplex centroid design}

A simplex centroid design with axial points in a pseudocomponent representation was used to optimize mobile phase composition. The simplex centroid design is a boundary point design. The prediction about mixture properties can appropriately describe if more runs in the interior of the simplex. Hence, the usual simplex design was augmented by the axial runs. Additionally, the overall centroid was augmented because it was not a design point.

The polynomial equations generated from experimental design were validated by ANOVA and $F$ statistics. ANOVA result and lack of fit tests of the models for all the responses are shown in Table 4. It has also indicated significant effects of the independent factors $(p>F)$ on response $Y_{1}, Y_{2}$, and $Y_{3}$. The larger $F$-value recommends that the data fit to the model which were significant and leads to good correlation with high $R^{2}$ value. For all the responses, adjusted and predicted $R^{2}$ values were in reasonable agreement, which suggests that the mathematical model describes the data adequately. However, certain model terms for $Y_{1}, Y_{2}$, and $Y_{3}$ having $p>0.05$ require model reduction in order to improve the model. Removal of these insignificant terms improved the model for $Y_{1}$ and $Y_{2}$. Although in the case of $Y_{3}$, removal of all the three insignificant terms, the model was not hierarchical, and therefore $X_{1} X_{2}$ was replaced back in the model.

The $F$ statistics in portion was used to test the generated reduced model. It shows that the $F_{\mathrm{Tab}}$ was greater than the $F_{\mathrm{Cal}}$ for all the responses indicating that the reduced term $\left(Y_{1}: \beta_{1233}, Y_{2}\right.$ : $\beta_{23}, Y_{3}: \beta_{1233}$ ) does not contribute significantly to the prediction of responses. Therefore, it was omitted from the full model (Table 4). Insignificant lack of fit for all responses also implies that the models were adequate for the prediction with the range of experimental variables. 
Table 7. Summary of validation parameters for the proposed HPLC method.

\begin{tabular}{|c|c|c|}
\hline \multicolumn{3}{|c|}{ Linearity } \\
\hline Linearity range (ng/ml) & \multicolumn{2}{|c|}{$10-500$} \\
\hline Correlation coefficient $\left(r^{2}\right)$ & \multicolumn{2}{|c|}{0.9993} \\
\hline Slop & \multicolumn{2}{|c|}{$166.54 \pm 1.63$} \\
\hline Intercept & \multicolumn{2}{|c|}{$7,642.43$} \\
\hline Regression equation & \multicolumn{2}{|c|}{$y=166.54 x+742.43$} \\
\hline \multicolumn{3}{|c|}{ Sensitivity } \\
\hline LOD (ng/ml) & \multicolumn{2}{|c|}{1.949} \\
\hline LOQ (ng/ml) & \multicolumn{2}{|c|}{5.907} \\
\hline \multicolumn{3}{|l|}{ Precision (\%RSD) } \\
\hline Repeatability & Mean \pm SD & $\%$ RSD \\
\hline 50 & $8,980.83 \pm 80.23$ & 0.893 \\
\hline 100 & $17,713.6 \pm 199.21$ & 1.125 \\
\hline 250 & $41,110.5 \pm 689.16$ & 1.676 \\
\hline \multicolumn{3}{|c|}{ Intraday } \\
\hline 50 & $8,989 \pm 75.44$ & 0.839 \\
\hline 100 & $\begin{array}{c}17,726.07 \pm \\
178.37\end{array}$ & 1.006 \\
\hline 250 & $41,139 \pm 631.17$ & 1.534 \\
\hline \multicolumn{3}{|c|}{ Inter day } \\
\hline 50 & $8,972.67 \pm 78.92$ & 0.88 \\
\hline 100 & $\begin{array}{c}17,686.96 \pm \\
217.91\end{array}$ & 1.232 \\
\hline 250 & $\begin{array}{c}41,032.11 \pm \\
576.31\end{array}$ & 1.405 \\
\hline \multicolumn{3}{|c|}{ Accuracy } \\
\hline Level & Mean \pm SD & $\%$ RSD \\
\hline 80 & $98.97 \pm 0.83$ & 0.841 \\
\hline 100 & $100.14 \pm 0.96$ & 0.963 \\
\hline 120 & $100.48 \pm 0.32$ & 0.314 \\
\hline
\end{tabular}

Table 8. Robustness study.

\begin{tabular}{lccccc}
\hline \multirow{2}{*}{ Parameters } & \multirow{2}{*}{$\begin{array}{c}\text { Normal } \\
\text { condition }\end{array}$} & \multirow{2}{*}{$\begin{array}{c}\text { Change in } \\
\text { condition }\end{array}$} & & \multicolumn{3}{c}{ \%RSD } \\
\cline { 6 - 6 } & 1 & \pm 0.2 & 1.446 & 1.601 & 1.786 \\
Flow Rate (ml/minute) & 3 & $\pm 0.2 \mathrm{pH}$ & 1.136 & 0.454 & 1.526 \\
$\mathrm{pH}$ & & & & & \\
Mobile phase ratio & $43: 55: 02$ & $\pm 1 \%$ & 1.772 & 1.226 & 1.811 \\
(ACN:H $\left.{ }_{2} \mathrm{O}: \mathrm{THF}\right)$ & & & & & \\
Extraction time (minute) & 15 & \pm 5 & 0.315 & 0.845 & 0.191 \\
\hline
\end{tabular}

RSD indicates: relative standard deviation; RT: retention time; TP: theoretical Plates; CAN: Acetonitrile; THF: tetrahydrofuran.

Apositive sign in polynomial equation favored relationship between the factor and the response while a negative sign showed the diminish effect on response. One can draw conclusion from the mathematical model itself if the main terms are significant. Direct interpretation of reduced polynomial equations may lead to errors since interaction and polynomial terms were also significant. Therefore, contour and response surface plots were drawn. Nonlinear relationship is visible in all contours and 3D surface plots (Fig. 3). Design space was identified based on the highest and the lowest range of variables. These plots were helpful in constituting desired responses and mixture compositions. In two-dimensional view of

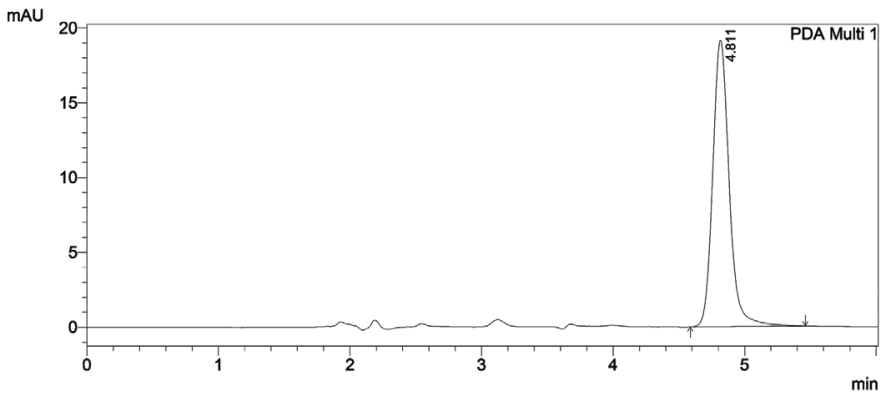

Figure 5. Validated experimental chromatogram.

the contour plots, constant responses were connected to construct the contour line. On the other hand, a 3D view of the surface plot served clearer picture of the response surface.

From the contour plot of RT (Fig. 3), the zone located toward the $X_{2}$ shows the maximum response. The proportion of aqueous phase (water) plays an important role in the mixture for delaying the RT. This may be due to increase in mobile phase polarity by water (Hao et al., 2008). In case of TF (Fig. 3), desired response was observed toward the vertices of $X_{1}$ and $X_{3}$. This indicated that ACN and THF component in the mixture are responsible for decreasing the TF. However, the content of THF in the mobile phase mixture was significantly responsible for minimizing the TF. Furthermore, Figure 3 shows the negative effect of $X_{1}$ on TP, whereas the addition of $X_{2}$ aids to improve the TP. At certain extent $X_{3}$ also contribute to the improvement of TP. This effect may be attributed to the $\mathrm{pH}$ of the water, which contributed to minimizing the ionization of elute. The overlaid contour plot can be used to select specific regions were all the mixture components are within optimum values.

The desirability function approach was employed to scrutinize the optimized mobile phase composition with desired responses. The optimized mobile phase composition (OC) with near-to-one desirability demonstrating its effectiveness in reaching the desired targets. The experimental design was validated by checkpoint analysis. The low $\%$ bias $(<10 \%)$ shows reasonable agreement between predicted and experimental values of OC and VC (Table 5). The chromatogram of PTV using optimized mobile phase composition shows a sharp peak with desired RT, least tailing and high TP (Fig. 5). These demonstrate the importance of current modeling approach in the establishment of an optimal analytical method.

These results also suggest the success of experimental design along with desirability approach for mobile phase composition optimization.

\section{System suitability and method validation}

The RSD values for system suitability parameters were found to be $<2 \%$, indicating the suitability of the instrument for the developed method (Table 6). The correlation coefficient $\left(r^{2}=0.9993\right)$ of the PTV calibration curve indicates the excellent relationship between concentration and peak area. It was observed that the LOD and LOQ values obtained were lower for this method, probably because of the good sensitivity for PTV. The developed method was validated and the results show low RSD $(<2 \%)$ indicating a high degree of accuracy and precision. The identical 
spectrum was found for standard pure drug and drug extracted from formulation indicates the peak purity. The peak purity was found to be $99.99 \%$, which suggests that the developed method is specific. The $\% \mathrm{RSD}(<2 \%)$ for robustness study suggests that the selected factors (RT, PA, and TP) were unaffected by small variations in the parameters.

\section{CONCLUSION}

The results showed that the dependence of peak quality parameters on mobile phase composition. This mobile phase composition was successfully optimized using a simplex centroid mixture design with desirability approach. Additionally, the developed method with an optimized mobile phase (Acetonitrile: $3 \mathrm{pH}$ Water: Tetrahydrofuran, 43:55:2) is validated, less time-consuming, and user friendly for the quantification of PTV in bulk and tablet samples.

\section{ACKNOWLEDGMENTS}

The authors would like to thank Dr. R. Balaraman and Dr. A. K. Seth for providing essential technical assistance.

\section{FINANCIAL SUPPORT}

None.

\section{CONFLICT OF INTEREST}

The authors declare that they have no conflicts of interest.

\section{REFERENCES}

Akabari AH, Shah DR, Shah SA, Suhagia BN. Kinetic determinations of pitavastatin calcium by stability indicating HPTLC method. J Liq Chromatogr Relat Technol, 2015; 38:521-31.

Alves de Almeida A, Spacino Scarminio I. Statistical mixture design optimization of extraction media and mobile phase compositions for the characterization of green tea. J Sep Sci, 2007; 30:414-20.

Atkinson AC, Tobias RD. Optimal experimental design in chromatography. J Chromatogr A, 2008; 1177:1-11.

Beg S, Kohli K, Swain S, Hasnain MS. Development and validation of RP-HPLC method for quantitation of amoxicillin trihydrate in bulk and pharmaceutical formulations using Box-Behnken experimental design. J Liq Chromatogr Relat Technol, 2012; 35:393-406.

Berridge JC. Techniques for the automated optimization of HPLC separations. Trends Analyt Chem, 1984; 3:5-10.

Cano CB, Felsner ML, Bruns RE, Matos JR, Almeida-Muradian LB. Optimization of mobile phase for separation of carbohydrates in honey by high performance liquid chromatography using a mixture design. J Braz Chem Soc, 2006; 17:588-93.

Cornell JA. Experiments with mixtures: designs, models, and the analysis of mixture data: John Wiley \& Sons, 2011

Deng JW, Kim KB, Song IS, Shon JH, Zhou HH, Liu KH, Shin JG. Determination of two HMG-CoA reductase inhibitors, pravastatin and pitavastatin, in plasma samples using liquid chromatography-tandem mass spectrometry for pharmaceutical study. Biomed Chromatogr, 2008; $22: 131-5$.
Di B, Su M-X, Yu F, Qu L-J, Zhao L-P, Cheng M-C, He LP. Solidphase extraction and liquid chromatography/tandem mass spectrometry assay for the determination of pitavastatin in human plasma and urine for application to Phase I clinical pharmacokinetic studies. J Chromatogr B, 2008; 868:95-101.

Gomas AR, Ram PR, Srinivas N, Sriramulu J. Degradation pathway for pitavastatin calcium by validated stability indicating UPLC method. Am J Analyt Chem, 2010; 1:83.

Hao Z, Xiao B, Weng N. Impact of column temperature and mobile phase components on selectivity of hydrophilic interaction chromatography (HILIC). J Sep Sci, 2008; 31:1449-64.

Hasnain MS, Rao S, Singh MK, Vig N, Gupta A, Ansari A, Sen P, Joshi P, Ansari SA. Development and validation of LC-MS/MS method for the quantitation of lenalidomide in human plasma using Box-Behnken experimental design. Analyst, 2013; 138:1581-8.

ICH Guideline HT. Validation of analytical procedures: text and methodology Q2 (R1)(2005). 2010 [Online]. Available via http://www.ich. org/cache/compo/363-272-1.html (Accessed 10 October 2015).

Kajinami K, Takekoshi N, Saito Y. Pitavastatin: efficacy and safety profiles of a novel synthetic HMG-CoA reductase inhibitor Cardiovasc Ther, 2003; 21:199-215.

Kojima J, Fujino H, Yosimura M, Morikawa H, Kimata H Simultaneous determination of NK-104 and its lactone in biological samples by column-switching high-performance liquid chromatography with ultraviolet detection. J Chromatogr B Biomed Sci Appl, 1999; 724:173-80.

Kumar SN, Baghyalakshmi J. Determination and quantification of pitavastatin calcium in tablet dosage formulation by HPTLC method. Anal Lett, 2007; 40:2625-32.

Montgomery DC. Design and analysis of experiments. John Wiley \& Sons, 2008

Panchal HJ, Suhagia BN, Patel MM, Patel BH. Estimation of pitavastatin calcium in tablet dosage forms by column liquid chromatography and ultraviolet spectrophotometry. J AOAC Int, 2008; 92:158-64.

Patel B, Suhagia B, Patel C, Panchal H. A simple and sensitive HPTLC method for quantitative analysis of darunavir ethanolate tablets. JPC-J Planar Chromat, 2011; 24:232-5.

Rao PV, Baral SS. Experimental design of mixture for the anaerobic co-digestion of sewage sludge. Chem Eng J, 2011; 172:977-86.

Srinubabu G, Raju CA, Sarath N, Kumar PK, Rao JS. Development and validation of a HPLC method for the determination of voriconazole in pharmaceutical formulation using an experimental design. Talanta, 2007; 71:1424-9.

How to cite this article:

Ramani VD, Jani GK, Sen AK, Sailor GU, Sutariya VB. Development and validation of RP-HPLC method for pitavastatin calcium in bulk and formulation using experimental design. J Appl Pharm Sci, 2019; 9(10):075-083. 